\title{
COMMENT \\ Human intestinal lymphoid tissue in time and space
}

\author{
Jo Spencer ${ }^{1}$, Jacqueline H Y Siu ${ }^{2}$ and Lucia Montorsi ${ }^{3,4}$ \\ Mucosal Immunology (2019) 12:296-298; https://doi.org/10.1038/s41385-018-0120-6
}

\section{INTRODUCTION}

Observational studies of human tissues have contributed to the literature for many years. However, recent initiatives driving human sample collection for research, the acquisition of deep next-generation datasets that enable observational accuracy and multidisciplinary collaborations between mathematicians and biologists are enabling a new age of observational science. In this issue of Mucosal Immunology, Senda et al. $2018^{1}$ describe the quantification of T-cell subsets in intestinal lymphoid tissue throughout the gut throughout life and demonstrate the feasibility of developing algorithms to classify gut tissues that may be adapted in the future for diagnosis of pathology.

\section{A NEW MAP OF HUMAN GUT-ASSOCIATED LYMPHOID TISSUE} The maintenance of homeostatic equilibrium at the epithelial interface between internal tissues of the body and the gut lumen is achieved by chronic immune response to antigen sampled from the lumen, initiated in the organised gut-associated lymphoid tissue (GALT). Cells activated in GALT are imprinted with the capacity to home back to the gut before wide dissemination via the blood and selective intestinal homing. GALT thus regulates the specificity and scale of the intestinal immune response and modulates the contents of the gut, including the composition of the microbiota.

Unlike most laboratory animal species that have prominent GALT in the Peyer's patches that is visible as protrusions even from the outside of the bowel, human GALT is difficult to see without magnifying or processing the bowel. ${ }^{2}$ An early map of the distribution of GALT through the human small intestine was provided by JS Cornes in $1965{ }^{3}$ who washed, fixed and stained samples before using transillumination and quantification of GALT by eye. This study informed us that human GALT is concentrated in the ileum, is present before birth and that it increases in quantity until puberty before gradually declining over the following decades. Now, 53 years later in this issue of Mucosal immunology, Senda et al., $2018^{1}$ supersede this study for the first time with an exceptional description of the distribution of lymphoid tissue throughout the small intestines, colon and the mesenteric lymph nodes of cadaver tissue donors from 4 months to 87 years old. They use a combination of histological analysis of segments of bowel and flow cytometric analysis of isolated cells to identify regional differences in lymphoid tissue content, T-cell subset variation and challenge the predictive value of their findings.

Senda et al. $2018^{1}$ describe the changes in lymphoid tissue along the intestine that imply that the antigenic challenge reduces from small bowel to large bowel. Features that support this statement include an increased proportion of naive CD4 and CD8 $T$ cells and a reduction in effector memory CD4 and CD8 T cells from jejunum to colon, in samples classified as mucosa or GALT. This may seem counterintuitive because it is widely considered that the microbiota, that is concentrated in the colon where it thrives in anaerobic conditions forming a colonic bacterial 'bioreactor', is a driving force behind the intestinal immune response, including the production of $\operatorname{lgA}$. It is possible that the structure of colonic follicles may protect them from exposure to the lumen to some degree. ${ }^{4}$ Follicles of colonic GALT are unique in their structure amongst GALT by the concentration of most of their lymphoid content on the serosal side of the muscularis mucosa (Fig. 1). The B-cell zone protrudes through the muscularis to make contact with a narrow follicle-associated epithelium (FAE) that may be located relatively deep in the mucosal layer. This contrasts with GALT at other sites where the FAE is rather prominent. Thus, the bulk of the T-cell zone is largely separated from the overlying mucosal barrier, implying that colonic GALT may differ functionally from GALT in other locations. O'Leary and Sweeney ${ }^{4}$ used the term 'lymphoglandular structures' to describe colonic lymphoid tissue. They observed that in health, most colonic lymphoglandular structures lack germinal centres, though the frequency of germinal centres increases when patients have cancer. This contrasts with lymphoid tissue elsewhere in the gut that has exposed FAE and germinal centres as prominent features. Drawing on the observations of Senda et al., $2018^{1}$ and O'Leary and Sweeney ${ }^{4}$ together, we may need to reappraise our view of the colonic microbiota as a major driver of intestinal immune responses in health. In reality, the structure of the colonic mucosa may keep microbiota and lymphoid tissue apart very effectively, leaving colonic lymphoid tissue in a relatively unstimulated state. The microbiota in health may be regulated by $\lg \mathrm{A}$ with broad specificity rather than selective responses to individual bacterial species. $^{5}$

\section{REDUCTION OF LYMPHOID TISSUE WITH AGE, EXCEPT IN COLON}

Like Cornes $1965^{3}$ Senda et al., $2018^{1}$ identify that follicles of lymphoid tissue in the small bowel reduce in frequency with age and they observe T-cell depletion consistent with their observation of thymic atrophy. ${ }^{6}$ A remarkable observation by Senda et al., $2018^{1}$ is that in contrast, the frequency of lymphoid follicles in the colon appears to be maintained throughout life. Selective retention of colonic GALT may suggest a unique and critical function that does not decline with age. The majority of cases of inflammatory bowel disease involve the colon. Likewise, colon is the major site of intestinal epithelial malignancy. Having the

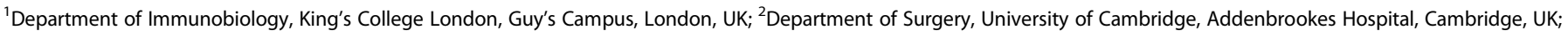
${ }^{3}$ School of Cancer Sciences, King's College London, Guy's Campus, London, UK and ${ }^{4}$ Cancer Systems Biology Laboratory, Francis Crick Institute, London, UK
} Correspondence: Jo Spencer (jo.spencer@kcl.ac.uk) 

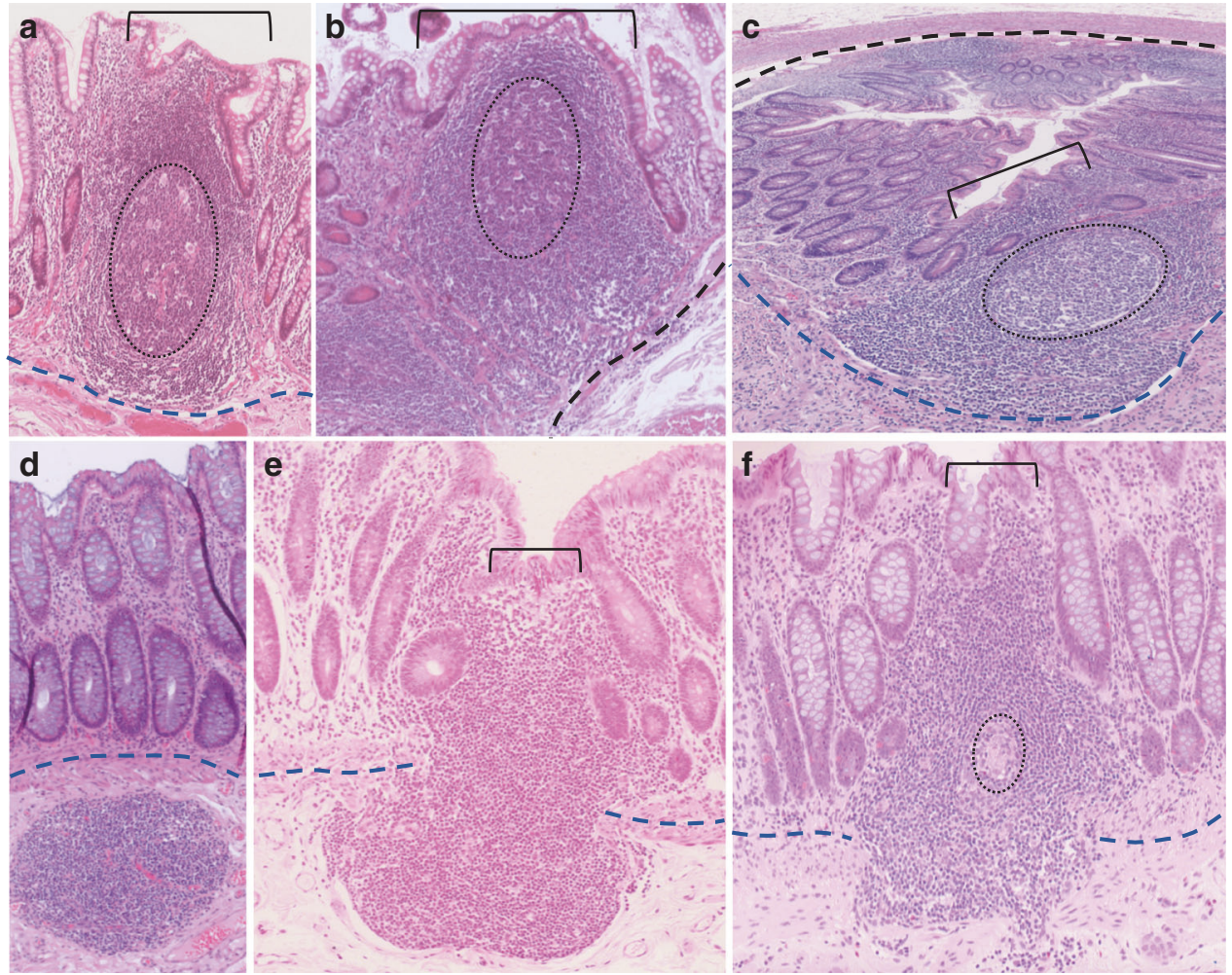

Fig. 1 Human GALT at different intestinal sites differs in position relative to the muscularis mucosa, the presence or not of germinal centres and the extent of the FAE. ${ }^{4}$ Paraffin sections of $\mathbf{a}$ and $\mathbf{b}$ small intestine, $\mathbf{c}$ transverse section through the appendix and $\mathbf{d}$-fcolon stained using haematoxylin and eosin. In each case, the dashed line indicates the position of the muscularis mucosae and brackets indicate the FAE. a Small intestinal isolated lymphoid follicles and $\mathbf{b}$ Peyer's patches are located on the mucosal aspect of the muscularis and have a FAE that is broadly exposed to the gut lumen. $\mathbf{b}$ Lymphoid tissue in the appendix (landscape view) is located on the mucosal side of the muscularis mucosa. The FAE tends to be less exposed than that of the Peyer's patches. $\mathbf{d - f}$ Lymphoid tissue in the colon is flask shaped comprising $\mathbf{d}$ a zone located on the serosal side of the muscularis mucosa, that in deeper sections e protrudes through the muscularis mucosa, and in $\mathbf{f}$ eventually forms a FAE that is narrow compared to GALT at more proximal sites in the gut. Whereas lymphoid tissue in the small intestine and appendix (a-c) invariably contains very large germinal centres (dotted circles) from an early age, GALT in the colon often has small or absent germinal centres $(\mathbf{d}-\mathbf{f})^{4}$

ability to protect the colon or the consequences of colonic mucosal breach may be a lifelong priority, but seemingly one that is mostly quiescent and rarely called on.

\section{OBSERVATIONAL IMMUNOLOGY COMES OF AGE}

The data presented by Senda et al., $2018^{1}$ have sufficient strength to enable prediction of sample donor age category in most cases and to reliably identify that a sample was derived from either GALT or mesenteric lymph node, though prediction of other site classifications was less robust. Nevertheless, in principle, this study represents an important step towards the goal of defining the baseline features of the human intestinal immune system against which changes in pathology can be measured. Some variability in data, particularly flow analysis of isolated cells is possibly due to the difficulty in reliably knowing the relative contributions of organised lymphoid tissues and lamina propria in intestinal isolates classified as mucosa. This issue has plagued this field for many years. ${ }^{2}$ The possibility that sample sites may be variably mixed in the GALT and lamina propria content may contribute to the lower success of predicting mucosal sites sampled.

It is an exciting time in human immunology when observational science is driven by the need to understand human immune physiology to maximise the capacity of personalised medicine. Whilst the study of Senda et al., $2018^{1}$ demonstrates the potential breadth of data across the intestinal immune system, developing technologies including immune repertoire sequencing, single-cell transcriptomics, proteomics, mass cytometry and imaging mass cytometry are also permitting depth of analysis at individual sites. The section of a normal human appendix in Fig. 2 has been stained using a panel of 29 rare-earth metal-tagged antibodies and analysed by imaging mass cytometry. Not only do these images permit histological examination as illustrated here, but they can be analysed through single-cell segmentation that can be enhanced by machine-learning algorithms. Segmentation would allow multidimensional cell data to be uploaded to analytical software, similarly to traditional flow cytometry data. In addition, since the electronic images comprise assemblies of pixels, these data can be handled as purely mathematical entities, creating a possible new generation of human tissue assessment strategies. $^{7-9}$

In the near future, when the depth of analysis and breadth of sampling are effectively combined in health and disease states, we anticipate that we will enter an era of molecular pathology where changes in the mucosa in disease states will be quantifiable against standard numeric parameters adjusted for age and gender. In this case, the relatively quiescent state of the colon at baseline may prove to be of particular value when considering the relevance of signature changes in inflammatory bowel disease and also cancer where colonic GALT activation has already been noted. ${ }^{4}$

\section{ACKNOWLEDGEMENTS}

This work was funded by the Medical Research Council of Great Britain (MR/L009382/ 1), the Gates Cambridge Trust, King's Health Partners and Cancer Research UK KHP 

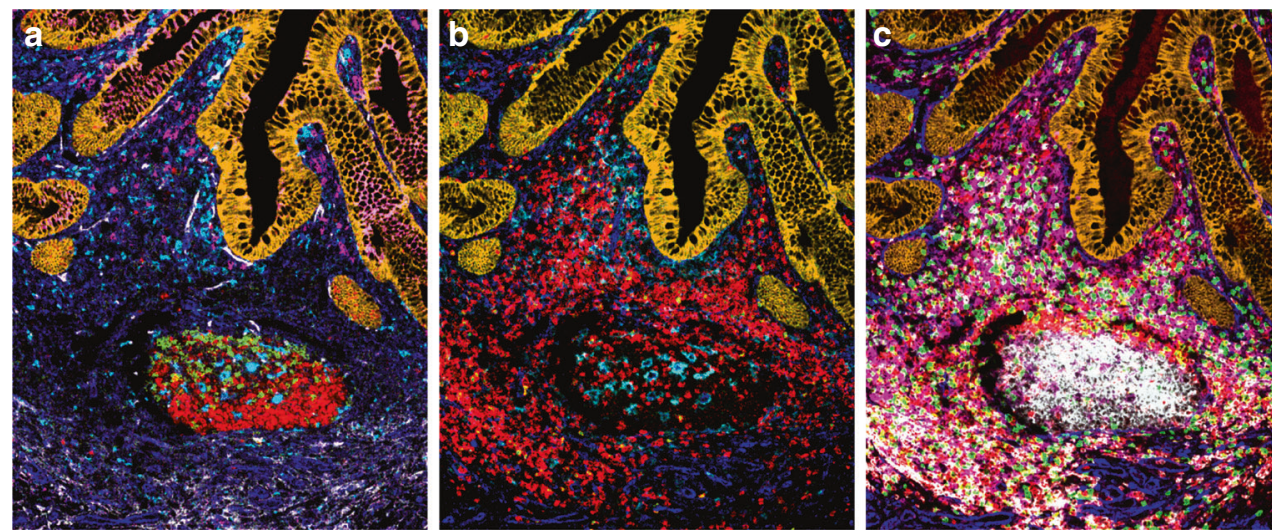

Fig. 2 Images of a paraffin section of a normal human appendix acquired with a Hyperion ${ }^{\mathrm{TM}}$ Imaging System (Fluidigm) and processed in histoCAT software. ${ }^{10}$ The distribution of 16 antigens is illustrated in three representations of the same region of interest. Antigens identified are a Ki67 (proliferation, red), PD1 (green), IgA (magenta), CD68 (macrophages, cyan), CD34 (endothelium, white), vimentin (dark blue) and Ecadherin (epithelium, orange). b CD3 (red), CD11c (cyan), PDL1 (dark blue), Foxp3 (green) and E-cadherin (orange). c CD45RA (red), CD4 (magenta), CD8 (green), CD20 (white), smooth muscle actin (dark blue) and E-cadherin (orange)

Cancer Centre. We are grateful to Professor Francesca Ciccarelli and Drs. Susanne Heck, Richard Ellis, Katrina Todd and Nedyalko Petrov of the National Institute for Health Research (NIHR) Biomedical Research Centre based at Guy's and St Thomas' NHS Foundation Trust for collaborating in the development of imaging mass cytometry.

\section{AUTHOR CONTRIBUTIONS}

J.S. and J.H.Y.S. wrote the manuscript. L.M. stained sections and created images for the figures. J.S. and L.M. assembled the figures.

\section{ADDITIONAL INFORMATION}

Competing interests: The authors declare no competing interests.

\section{REFERENCES}

1. Senda et al.Microanatomic dissection of human intestinal immunity reveals site specific changes in gut-associated lymphoid tissues over life. (2018)
2. Spencer, J. \& Sollid, L. M. The human intestinal B-cell response. Mucosal Immunol. 9, 1113-1124 (2016).

3. Cornes, J. S. Number, size, and distribution of Peyer's patches in the human small intestine: part I the development of Peyer's patches. Gut 6, 225-229 (1965).

4. O'Leary, A. D. \& Sweeney, E. C. Lymphoglandular complexes of the colon: structure and distribution. Histopathology 10, 267-283 (1986).

5. Rollenske, T. et al. Cross-specificity of protective human antibodies against Klebsiella pneumoniae LPS O-antigen. Nat. Immunol. 19, 617-624 (2018).

6. Kumar, B. V., Connors, T. J. \& Farber, D. L. Human T cell development, localization, and function throughout life. Immunity 48, 202-213 (2018).

7. Zhao, Y. et al. Spatiotemporal segregation of human marginal zone and memory B cell populations in lymphoid tissue. Nat. Commun. 9, 3857 (2018).

8. Keren, L. et al. A structured tumor-immune microenvironment in triple negative breast cancer revealed by multiplexed ion beam imaging. Cell 174, 1373-1387 e1319 (2018).

9. Bodenmiller, B. Multiplexed epitope-based tissue imaging for discovery and healthcare applications. Cell Syst. 2, 225-238 (2016).

10. Schapiro, D. et al. histoCAT: analysis of cell phenotypes and interactions in multiplex image cytometry data. Nat. Methods 14, 873-876 (2017). 